وقائع المؤتمر العلمي الحادي عشر- كلية الطب البيطري 43- 49: 2012

الاداء الفسيولوجي للمواليا المرضعة من امهات معاملة بمستويات مختلفة من تنكستات

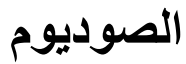

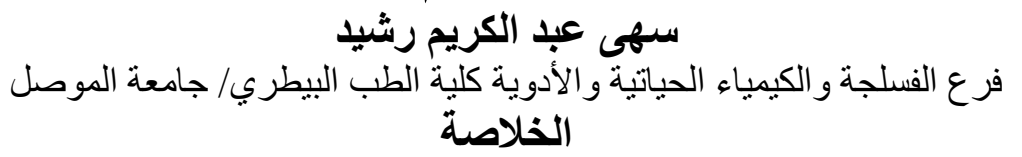

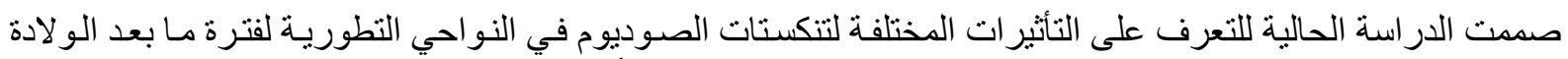

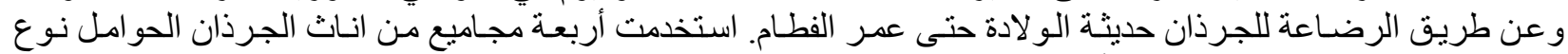

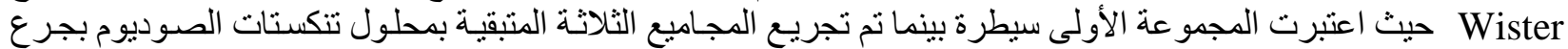

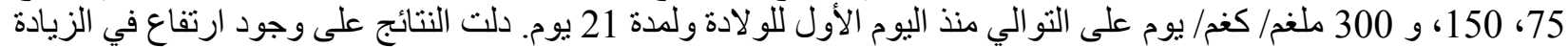

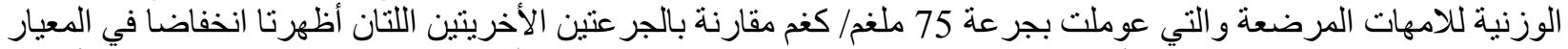

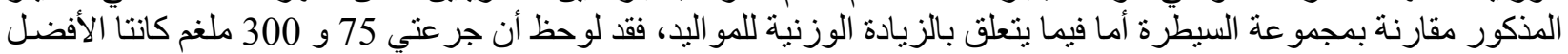

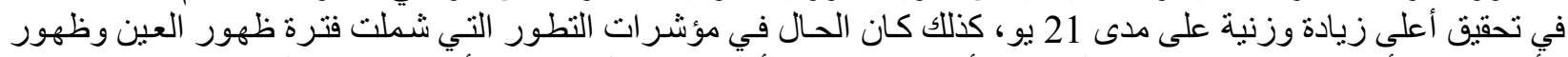

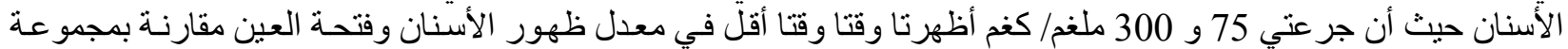

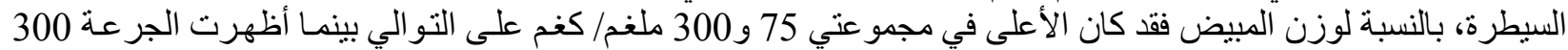

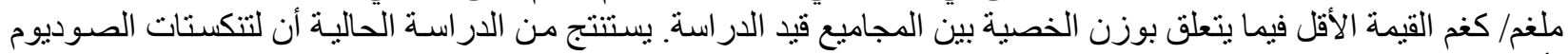

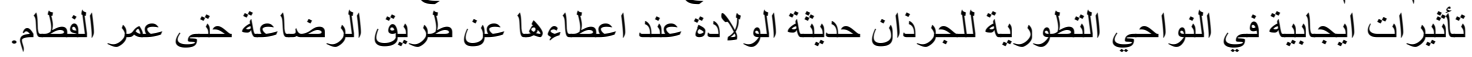

\title{
The physiological performance of offspring nursing from dams treated with different levels of sodium tungstate
}

\author{
Suha A. Rasheed \\ Department of Physiology, Biochemistry and Pharmacology, College of Veterinary Medicine, \\ University of Mosul \\ Mosul, Iraq \\ suharasheed@yahoo.com
}

\section{Summary}

This study aimed to recognize the different effects of administration of sodium tungstate in developmental indices of the offspring till weaning. Four groups of Albino Wistar pregnant female rats were used, the first one was control while the remaining three groups intubated with $\mathrm{Na}$ - tungstate 75,150 and $300 \mathrm{mg} / \mathrm{kg} /$ day respectively since the first day of parturition throughout 21 days. Results referred to an elevation in weight gain of rat dams treated with 75 $\mathrm{mg} / \mathrm{kg}$ while weight gain of offspring elevated in both doses of 75 and $300 \mathrm{mg} / \mathrm{kg}$ throughout 21 days, teeth and eye orifice were observed in doses of 75 and $300 \mathrm{mg} / \mathrm{kg}$ faster than control while the mean of ovary weight elevated in both 75 and $300 \mathrm{mg} / \mathrm{kg}$ however the dose $300 \mathrm{mg} / \mathrm{kg}$ revealed the less value of testes weight. The conclusion of present study is that Na- tungstate has positive effects on developmental indices in offspring when administered through nursing until weaning.

Key words: tungstate, developmental, offspring, rat.

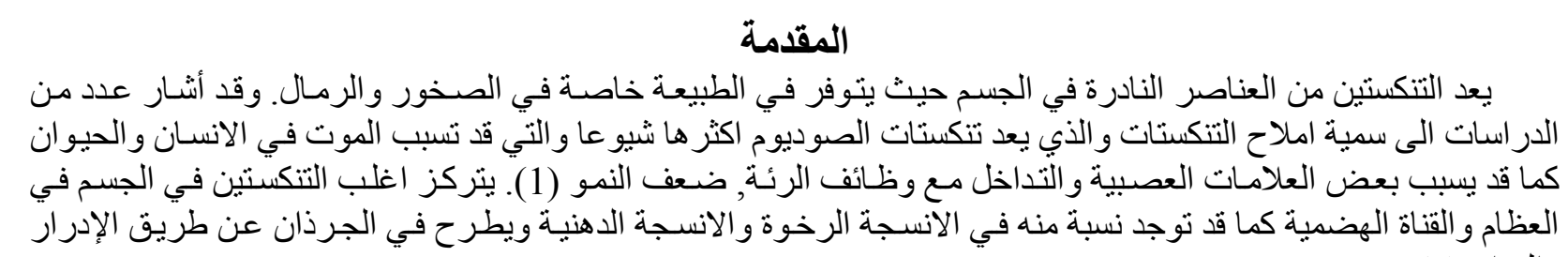
و البراز (1). 


\section{وقائع المؤتمر العلمي الحادي عشر- كلية الطب البيطري 43- 49: 2012}

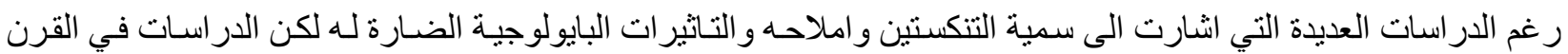

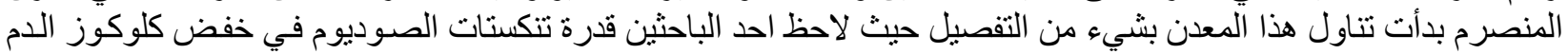

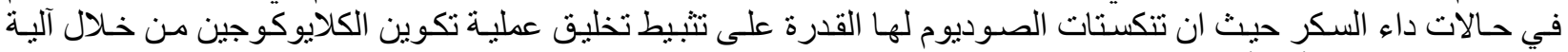

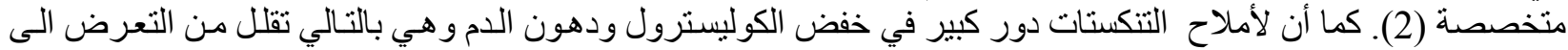

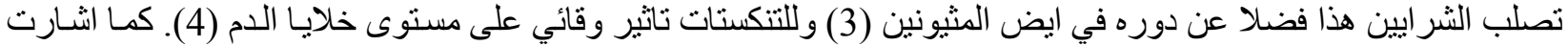

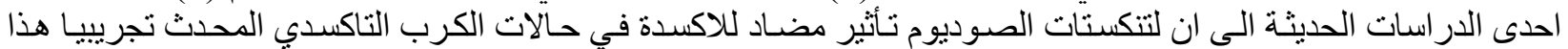

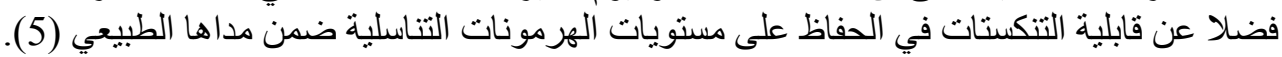

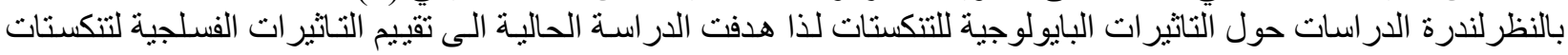

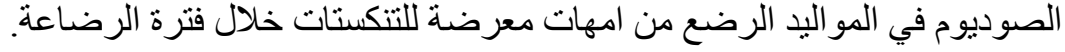

\section{المواد وطرق العمل}

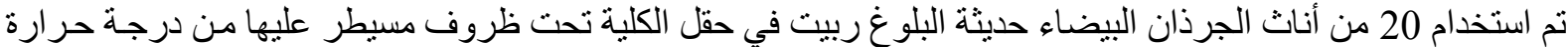

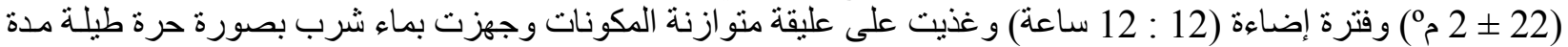

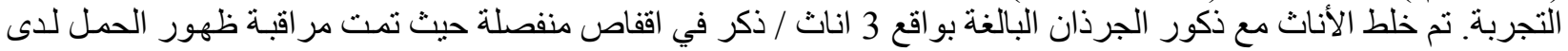

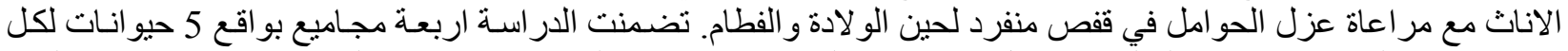

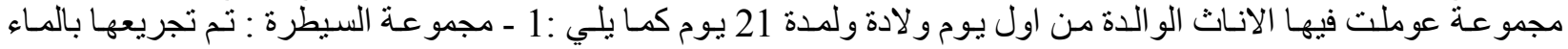

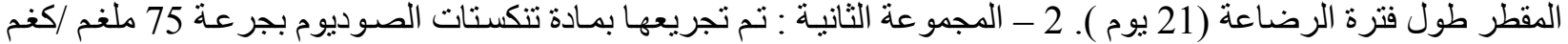

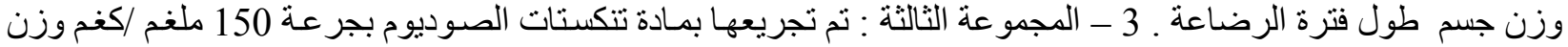

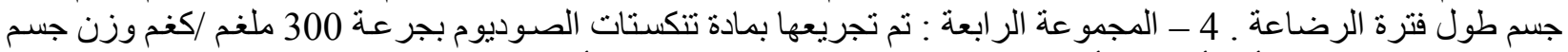

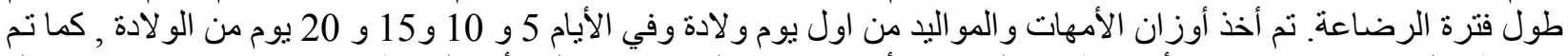

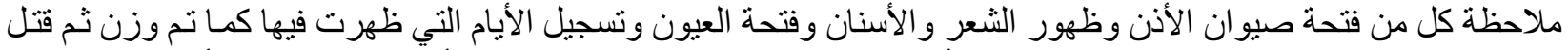
المو اليد بعمر 21 يوم وتم تسجيل اوز ان كل من الأعضاء التناسلية الذكرية ( الخصى ) و الأعضـاء التناسلية الأنثويـة (المبايض

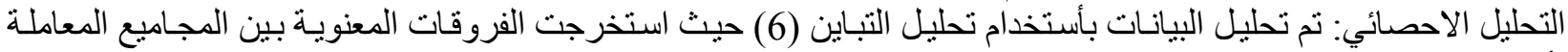

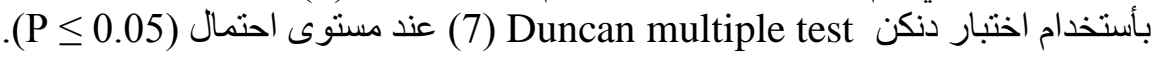

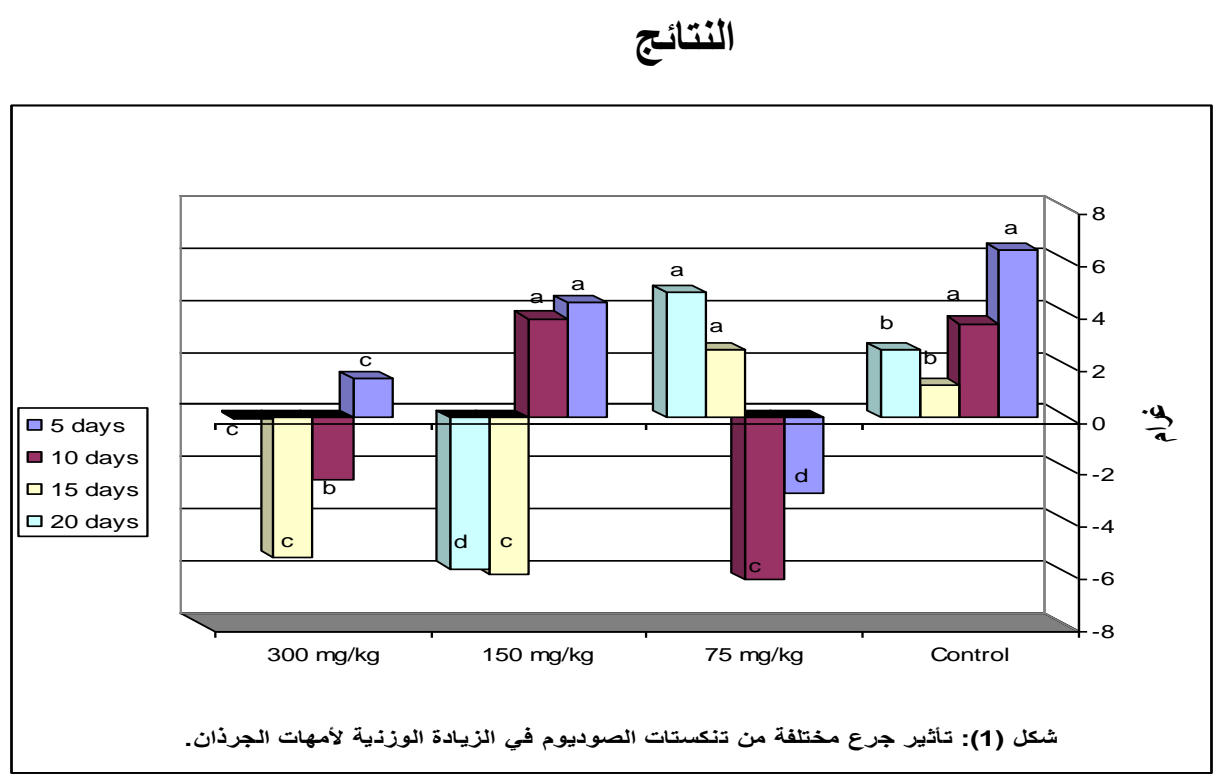

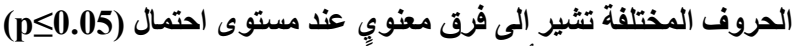

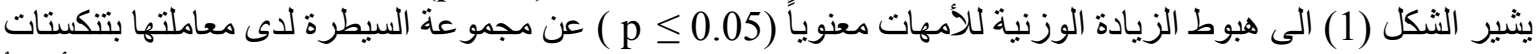

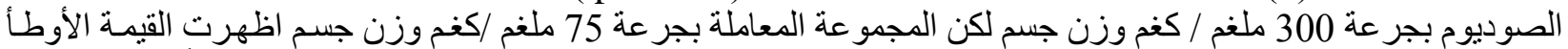

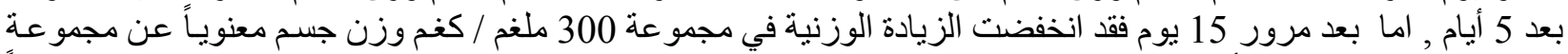

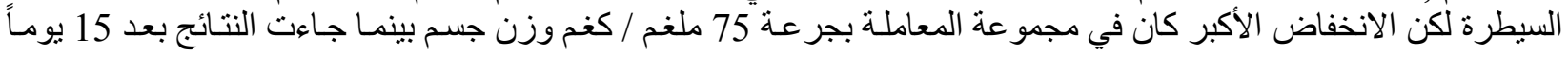




\section{وقائع المؤتمر العلمي الحادي عثر- كلية الطب البيطري 43- 49: 2012}

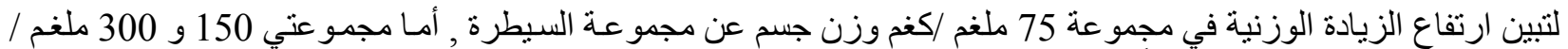

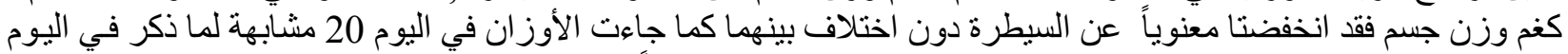

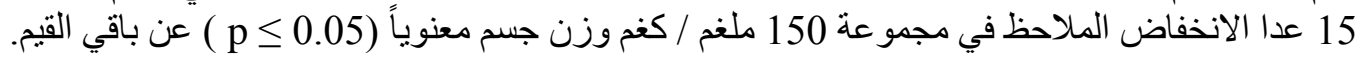

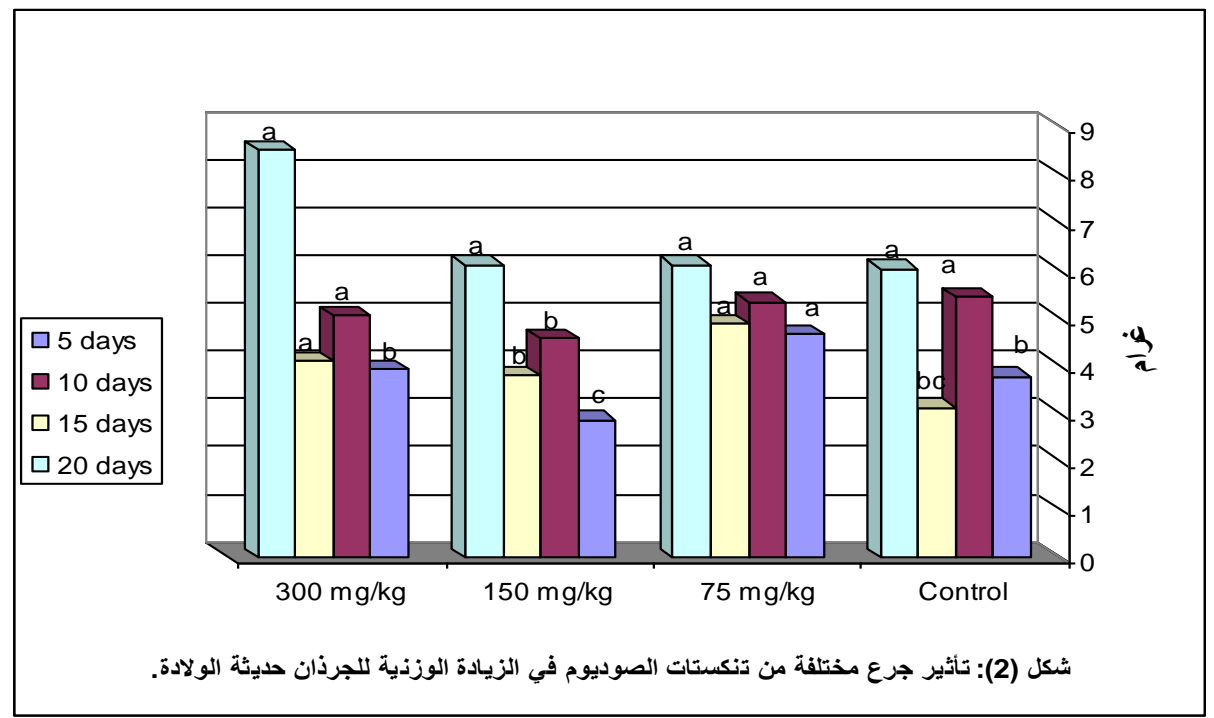

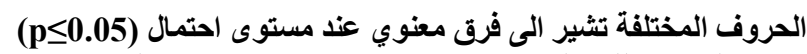

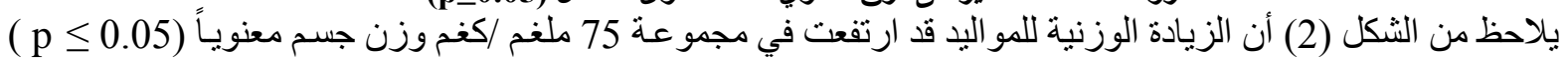

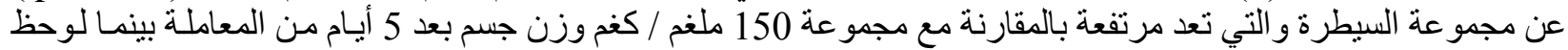

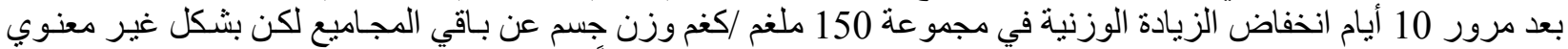

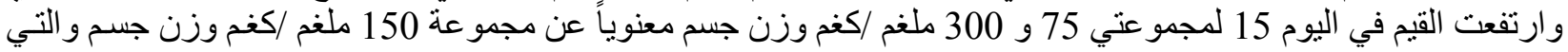
لم تختلف عن السيطرة. كما ارتفعت القيم بعد 20 يو في في كل المجاميع بحيث لم يظهر بينها اختلاف معنوي.

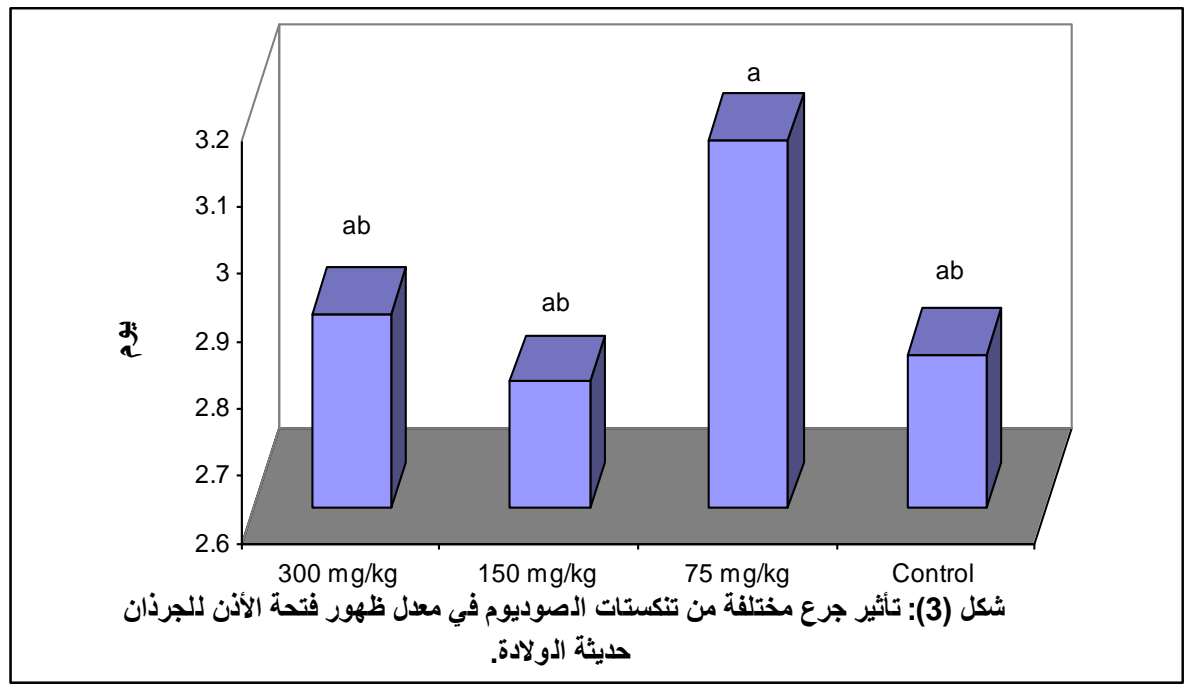

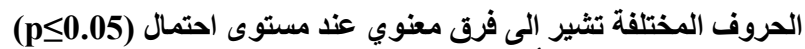

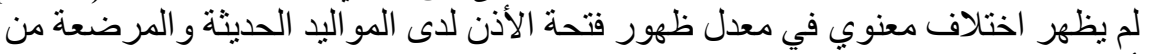
قبل أمهات معاملة بجرع من تنكستات الصوديوم بالمقارنة مع السيطرة (شكل 3 ) ). وكذللك الحال في معدل ظهور الثـعر (شكل 
وقائع المؤتمر العلمي الحادي عشر- كلية الطب البيطري 43- 49: 2012

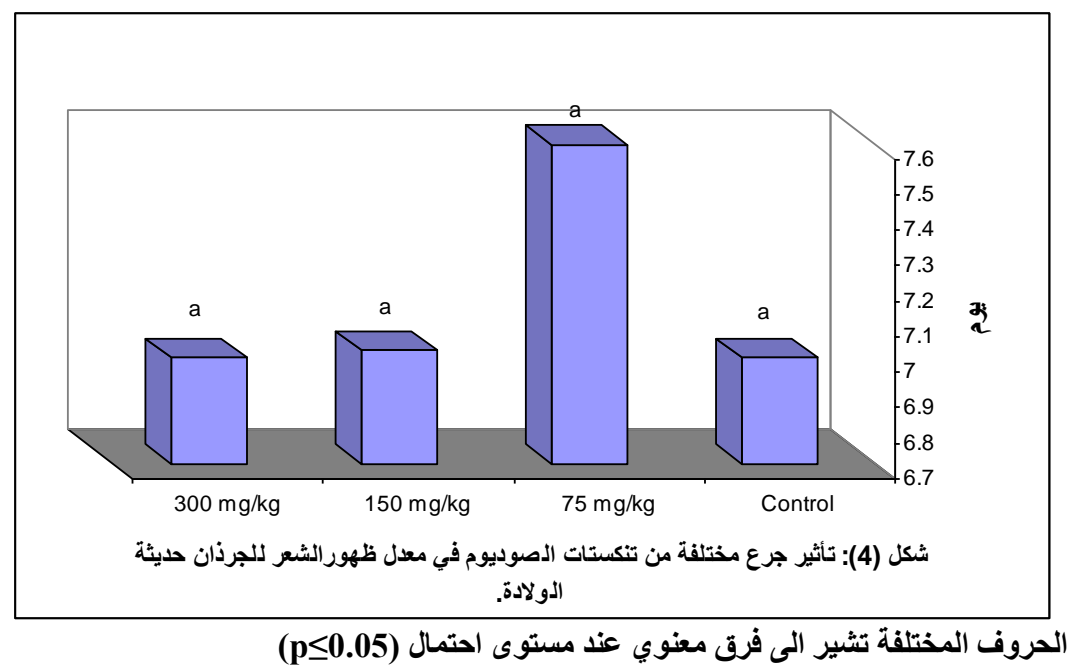

لقد أظهرت مجمو عة 150 ملغم / كغم وزن جسم بعض السر عة في ظهور الأسنان بالمقارنـة مـع السيطرة و المجمو عتين

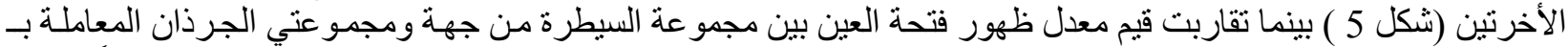

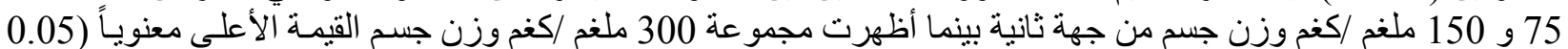
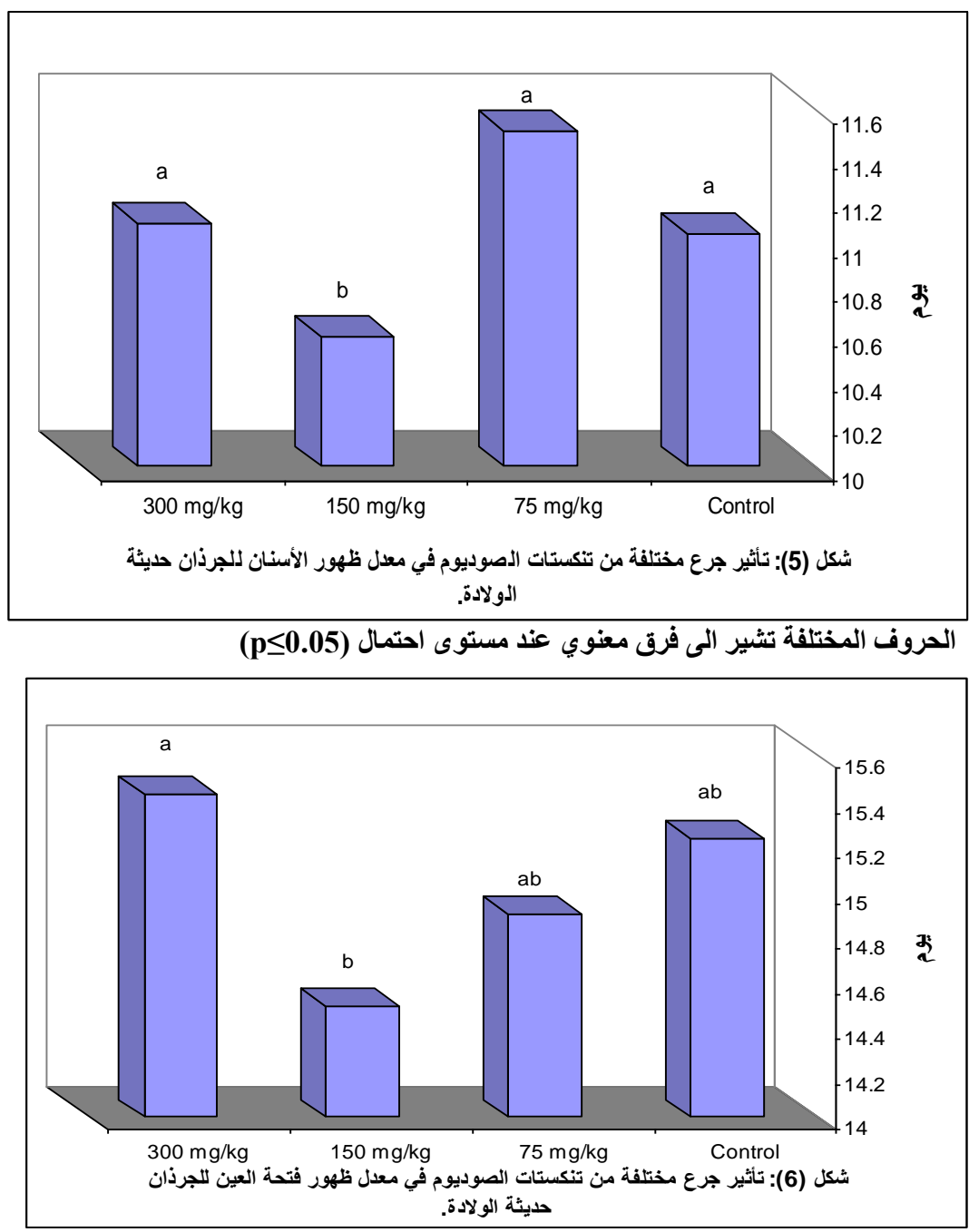

الحروف المختلفة تثير الى فرق معنوي عند مستوى احتمال (p 


\section{وقائع المؤتمر العلمي الحادي عثر- كلية الطب البيطري 43- 49: 2012}

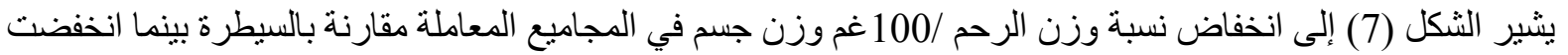

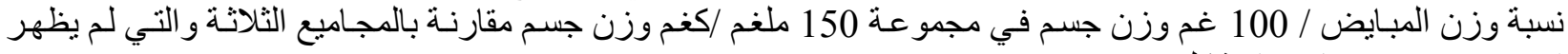

اختلاف معنوي فيما بينها (شكل 8) الشبان
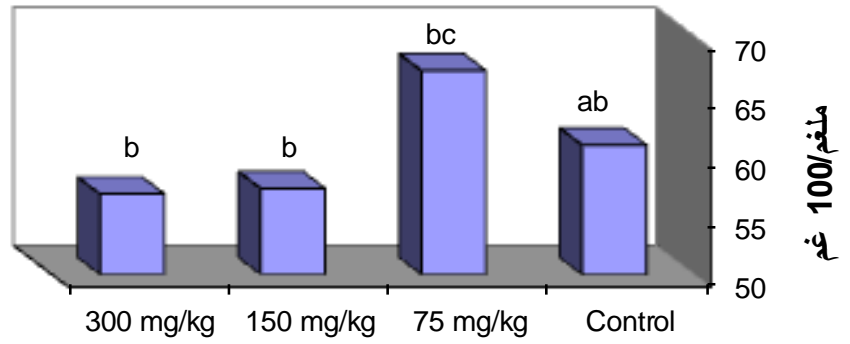

شكل (7): تأثير جرع مختلفة من تنكستات الصوديوم في معدل وزن الرحم للجرذان حديثة الولادة.

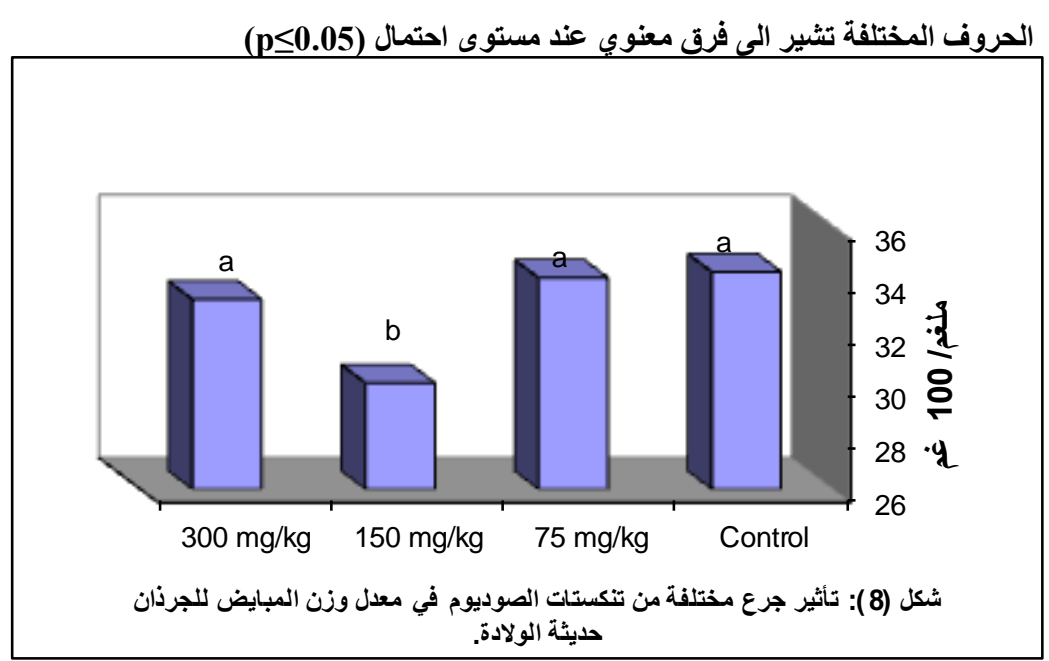

الحروف المختلفة تثبر الى فرق معنوي عند مستوى احتمال (p0.05)

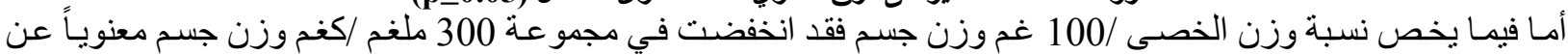

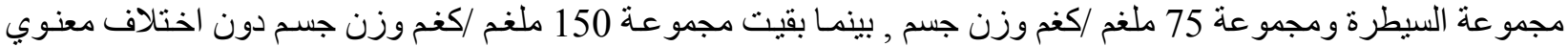
عن مجمو عة السيطرة و 75 ملغم /كغم وزن جسم من جهة ومجمة ولمو عة 300 ملغم /كغم وزن جسم من جهة ثانية ( شكل 9 ).

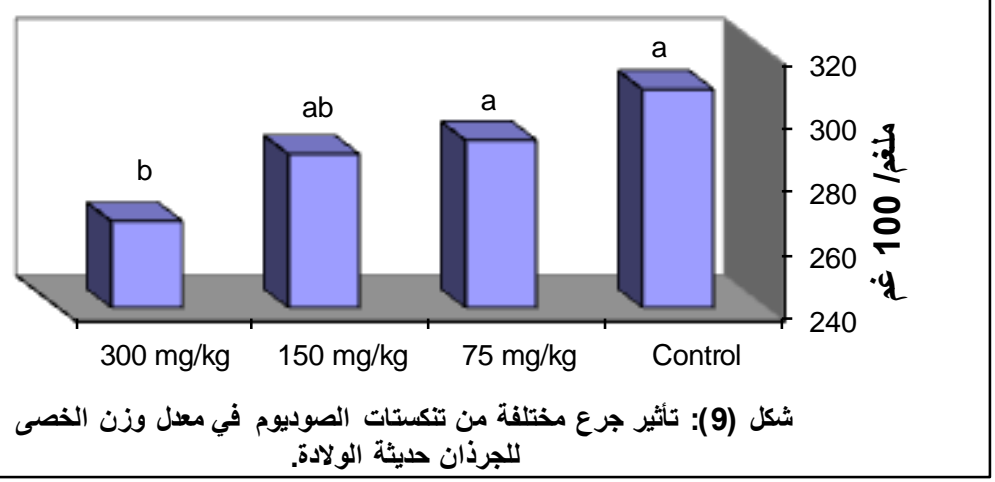

الحروف المختلفة تثبر المى فرق معنوي عند مستوى احتمال (p0.05) 


\section{وقائع المؤتمر العلمي الحادي عشر- كلية الطب البيطري 43- 49: 2012}

\section{المناقشة}

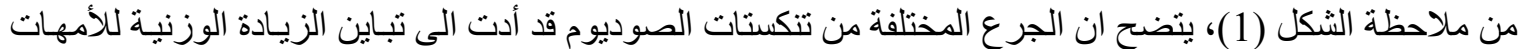

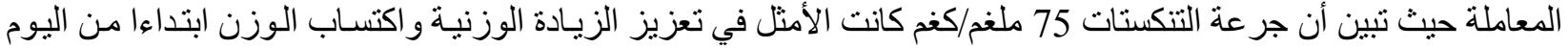

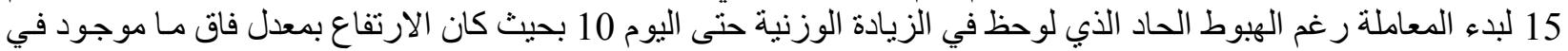

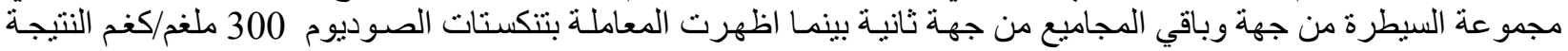

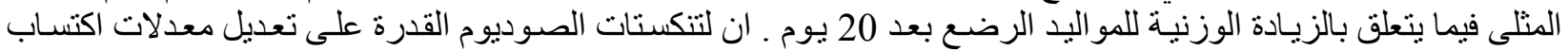

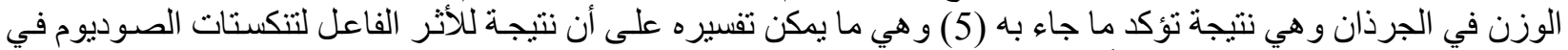

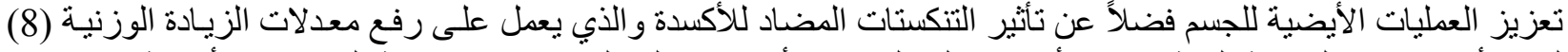

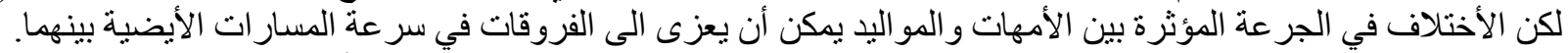

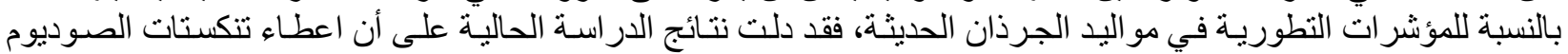

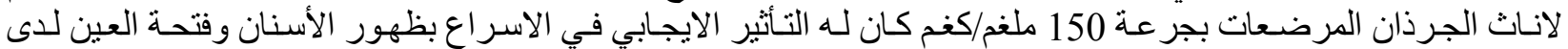

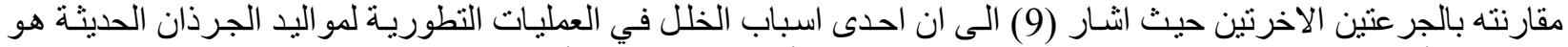

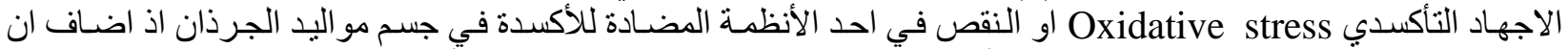

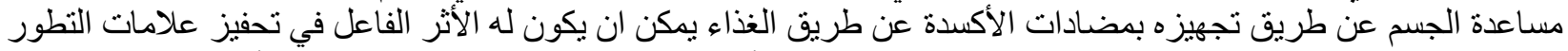

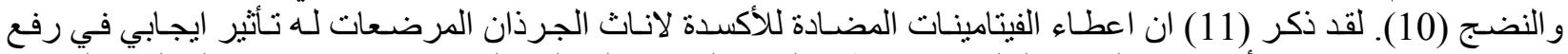

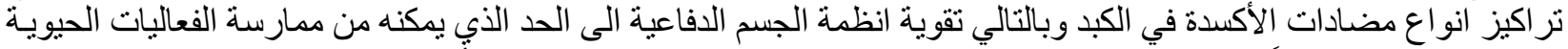

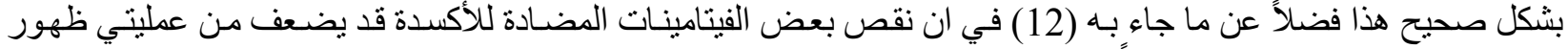

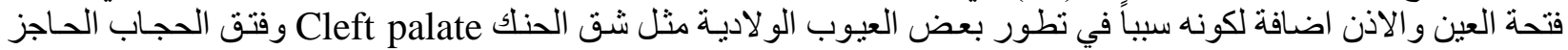

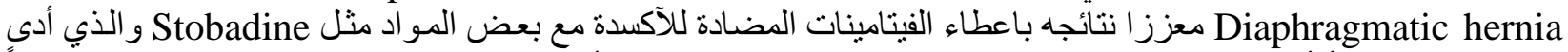

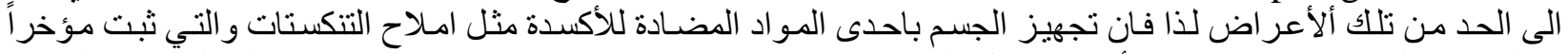

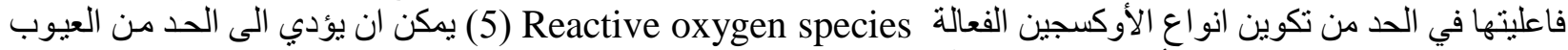

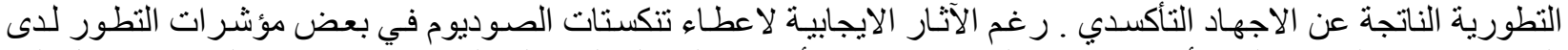

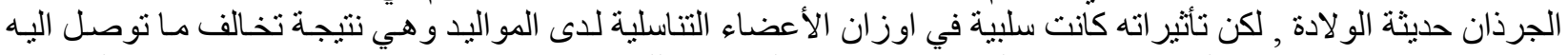

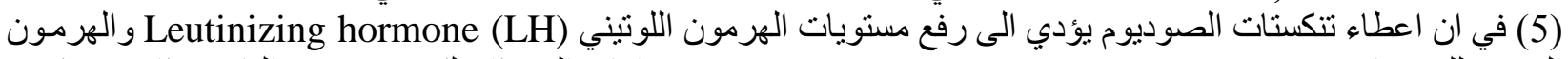

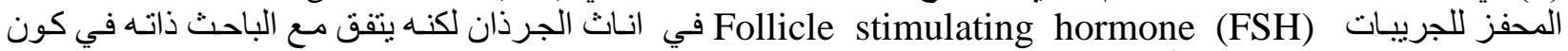

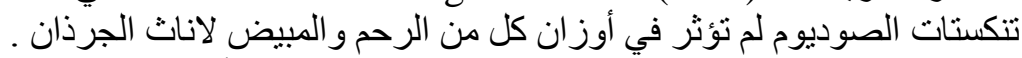

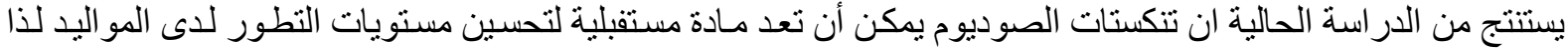

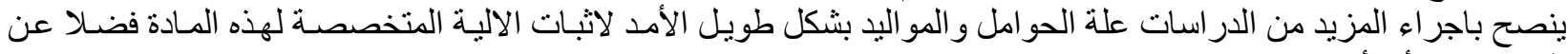
التحري عن أية أعر اض جانبية.

\section{شكر وتقدير}

تتقدم الباحثة بالثكر الى كلية الطب البيطري لدعمها الدر اسة كما نتقلدم بالامتنان الى الأستاذ المسـاعد نـاظم أحمد حسن لتقديمهـ المساعدة و المشورة العلمية.

\section{المصادر}

1.National Research Council NRC (1980). Tungsten .In: Mineral Tolerance of Domastic Animals. National Academy of Sciences, Washington.

2.Munoz MC Babera A Domingue J Fernandez - Al varez $\mathrm{J}$ Gomis $\mathrm{R}$ and Guinovart $\mathrm{JJ}$ (2001). Effect of tungstate, a new potential oral antidiabetic agent, in Zucker diabetic fatty rats. Diabetes, 50: 131 -138.

3.Tagagi S Nakajima S Fukuo $\mathrm{Y}$ and Terashi A (1998). Influences of Supplementary dietary tungsten on methionine metabolism in Rabbits fed a low - cholesterol plus methionine diet. J. Atheroscler. Thromb., 5 (1): 13 - 20.

4.Sato K Ichimasa M Shiomi M Nishimura Y and Ichimasa Y. (1999). Radioprotective effects of sodium tungstate on hematopoie tic injury by exposure to 60 Co gamma - rays in wistar rats . J. Radiat. Res. (Tokyo), 40(2): 101-113. (Abstract).

5.رشيد ' سهى عبد الكريم (2011 ) تأثير استخدام تنكستات الصوديوم في بعض الصفات الفسلجية للجرذان المعرضة للكرب

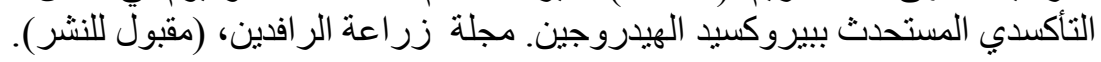


6. Steel RGD and Torrie JH (1980). Principle and Procedures of Statistics. $2^{\text {nd }}$ ed. New York. Mc-Graw-Hill Book Company Inc.

7.Duncan DB (1955). Multiple range and multiple " F" test. Biometric 11: 1-42.

8. Nakhaee A Bokaeian M Akbarzadeh A and Hashemi M (2010).Sodium tungstate Attenuate oxidative stress in brain tissue of streptozotocin- induced diabetic Rats. Biol. Trace. Elem. Res.,136(2): 221-231.

9.Sarbattama S and Rebecca AS (2010). Maternal anti0xidant supleme ntation prevents adiposity in the offspring of western diet - fed rats .Diabetes, 59: 3058-3065.

10.Jonathan MD and Richard LA (2010). Maturation of the antioxidant system and the effects on preterm birth. Seminars in fetal and neonatal medicine, 15: 191-195.

11.Ashley RV and Sherry AT (2005). One - time vitamin A supplem entation of lactating sows enhances hepatic retinol in their offspring independent of dose size. Am J Clin Nutr., $81(2) 427-433$.

12. Dubovickyi M Ujhzyi E Kovacovsk P Rychlk I Navarov J and Jansk J (1999). Antioxidant stobadine and neurobehavioural development of the rat offspring. Gen Physiol Biophys., 18: 4147. 